\title{
INFLUENCE DU DÉBIT DE BASE ET DE LA DURÉE DES ÉCLUSÉES SUR LA DÉRIVE D'ALEVINS DE TRUITE COMMUNE : EXPÉRIMENTATIONS EN CANAL SEMI-NATUREL.
}

\author{
H. LIEBIG, P. LIM et A. BELAUD
}

Laboratoire d'Ingénierie Agronomique - Equipe Environnement Aquatique et Aquaculture, INP-ENSAT, Avenue de l'Agrobiopole, BP 107 - Auzeville-Tolosane, 31326 Castanet-Tolosan Cedex, France.

\section{RÉSUMÉ}

Le fonctionnement par éclusées de certains ouvrages hydroélectriques installés sur des rivières de montagne à peuplement salmonicole est souvent considéré comme responsable du déficit en alevins observé sur les secteurs à l'aval immédiat du rejet. Une des causes majeures avancée de ce déficit est l'entraînement forcé des alevins dès l'émergence. Une approche expérimentale de ce phénomène est apparue nécessaire pour identifier et quantifier les facteurs mis en jeu. Un ancien bras de la rivière Oriège a été ainsi aménagé au niveau du canal de fuite de l'usine hydroélectrique d'Orlu, en Ariège (09). Ce système semi-naturel permet, sur un substrat et des abris naturels, de simuler des éclusées et de quantifier la dérive des alevins résultante. Sur un bief à faciès d'écoulement mixte radier-plat, nous avons testé deux modalités pour la durée des éclusées (10 minutes et 3 heures), associées à deux modalités pour la valeur du débit de base (débit précédant l'éclusée), soit 150 et $250 \mathrm{l} / \mathrm{s}$. Le débit plafond des éclusées a été fixé à $1500 \mathrm{l} / \mathrm{s}$, ce qui correspond à un facteur de 6 à 10 par rapport aux débits de base, situation fréquemment rencontrée sur l'Oriège.

Les résultats sont comparés et discutés. En définitive, il apparaît que les éclusées entraînent systématiquement une dérive forcée des alevins. Cette dérive intervient principalement au démarrage de l'éclusée et est d'autant plus forte que le débit de base est faible.

\section{INFLUENCE OF BASIC FLOW AND HYDROPEAKING DURATION ON THE DRIFT OF POST-EMERGENT FRY OF BROWN TROUT : EXPERIMENTS ON A SEMI-NATURAL STREAM.}

\begin{abstract}
In salmonid streams, hydropeaking management is often considered as responsible for the decrease in fry density observed downstream of water outlets. The fry carrying, by flow, could be one of the main causes of such a deficit. To identify and quantify factors involved, the old streambed of the Oriège stream (French Pyrenees) was arranged. In this semi-natural stream, with natural substratum and refuges, it is possible to simulate hydropeaking and to quantify the associated fry drift. On a section with riffle-glide sequence, two hydropeaking duration (10 min and 3 hours), and two basic flow values (the flow before the spate) : 150 and $250 \mathrm{l} / \mathrm{s}$ were tested. The spate flow was fixed at $1,500 \mathrm{l} / \mathrm{s}$, from 6 up to 10 times the basic flow, as it is frequently recorded on the Oriège river.
\end{abstract}


The results are compared and discussed. We concluded that hydropeakings cause a forced drift of post-emergent trout fry, which occurs at the beginning of hydropeaking. Moreover, this drift depended on the basic flow value.

\section{INTRODUCTION}

Parmi les ouvrages hydroélectriques implantés sur les cours d'eau français, 144 sont gérés par " éclusées ", turbinant par intermittence l'eau stockée dans des barragesréservoirs (LAUTERS, 1993). Ce mode de gestion permet d'utiliser l'énergie potentielle représentée par l'eau stockée, au moment où la demande d'électricité est forte. II se traduit, à l'aval des installations, par des variations brutales de débit, à court ou très court terme.

Pour déterminer l'influence de ces fluctuations artificielles de débit, souvent jugées responsables d'impacts négatifs sur la faune aquatique (CUSHMAN, 1985 ; LAUTERS, 1992 ; CEREGHINO, 1997), un programme national de recherche a été lancé par la Direction des Etudes et Recherches d'Electricité De France. Des travaux réalisés dans ce cadre ont mis en évidence un déficit important en alevins dans les populations de truites à l'aval d'ouvrages hydroélectriques gérés par éclusées (LAUTERS, 1995). Par la suite, une approche expérimentale préliminaire menée sur des alevins de truite de 2 à 3 mois, issus de pisciculture, a permis à cet auteur d'avancer l'hypothèse d'un entraînement forcé des alevins par ces mêmes éclusées, en insistant sur le rôle que pourrait jouer le débit de base. Par ailleurs, VALENTIN et al. (1994a) ont montré dans des situations comparables une réduction de la densité d'alevins d'ombre. Cependant, ces mêmes auteurs (VALENTIN et al., 1994b), n'ont pas montré, lors de simulations d'éclusées, d'augmentation significative de la dérive d'alevins d'ombre âgés de 2 mois, alors que les effets sur des alevins de truite du même âge apparaissaient modérés (VALENTIN, 1995).

D'autres travaux, sur la distribution spatiale et la survie dès l'émergence des alevins de diverses espèces de salmonidés, ont montré la fragilité des alevins émergents dans leur nouveau milieu (ELLIOTT, 1989 ; BARDONNET et al., 1993 ; GAUDIN et HELAND, 1995 ; HELAND et al., 1995), en particulier face aux variations de débit (OTTAWAY et CLARKE, 1981 ; IRVINE, 1986 ; HEGGENES et TRAAEN, 1988).

A partir de ces informations, il est apparu nécessaire d'une part de vérifier dans un cadre expérimental cohérent que les éclusées provoquent bien une dérive des alevins post-émergents de truite commune, et d'autre part de quantifier cet impact. Pour cela, 3 hypothèses majeures ont été définies: (1) que les éclusées sont responsables d'une dérive accentuée des jeunes alevins de truite, (2) que la valeur du débit de base conditionne cet effet en influant sur la réponse de l'alevin en termes de sélection et d'accès à l'abri, (3) que l'impact se produit principalement au démarrage de l'éclusée. Afin de les vérifier, nous avons dû mettre au point un système expérimental adapté, résolument orienté sur la reproduction de conditions d'environnement réelles (simulation d'éclusées importantes, travail sur un milieu proche de l'habitat naturel des alevins...) plutôt que sur des capacités d'observations comportementales directes.

Le but du travail présenté ici est de décrire le fonctionnement de cet outil et d'exposer les premiers résultats obtenus.

\section{MATÉRIEL ET MÉTHODES}

\section{Dispositif expérimental}

Les observations ont été réalisées sur le canal semi-naturel de l'Oriège, constitué par l'ancien lit de cette rivière, court-circuité lors de l'installation de l'usine hydroélectrique des Forges d'Orlu qui turbine l'eau d'un lac d'altitude (Figure 1). Long de près de $140 \mathrm{~m}$, le 
canal est alimenté conjointement par l'eau de l'Oriège et de la centrale hydroélectrique, ce qui confère aux éclusées simulées les mêmes caractéristiques physico-chimiques que les eaux réellement turbinées. Des vannes permettent un contrôle précis du débit entrant. Un large seuil jaugé situé à la moitié du parcours permet de vérifier le débit transitant. Le canal est divisé en 4 biefs de 26 à 40 mètres de longueur, dotés chacun d'un support permettant la fixation d'un filet de maille $2 \mathrm{~mm}$, barrant la totalité du lit et filtrant l'ensemble du débit. Le contenu du filet est transféré sur des tables de tri où sont récupérés et dénombrés les alevins dévalants. Le substrat a été conservé d'origine. Aucun apport de nourriture extérieure n'est nécessaire du fait de l'abondance d'invertébrés benthiques qui colonisent naturellement les biefs. De plus, du fait de l'alimentation par les eaux de l'Oriège, la dérive des invertébrés procure un apport de nourriture supplémentaire. Avant les expérimentations, le bief est pêché afin d'éliminer les poissons qui auraient pu remonter de l'aval. Les filets sont alors mis en place et fermés. Le débit de base est réglé à la valeur choisie, vérifiée grâce au seuil jaugé.

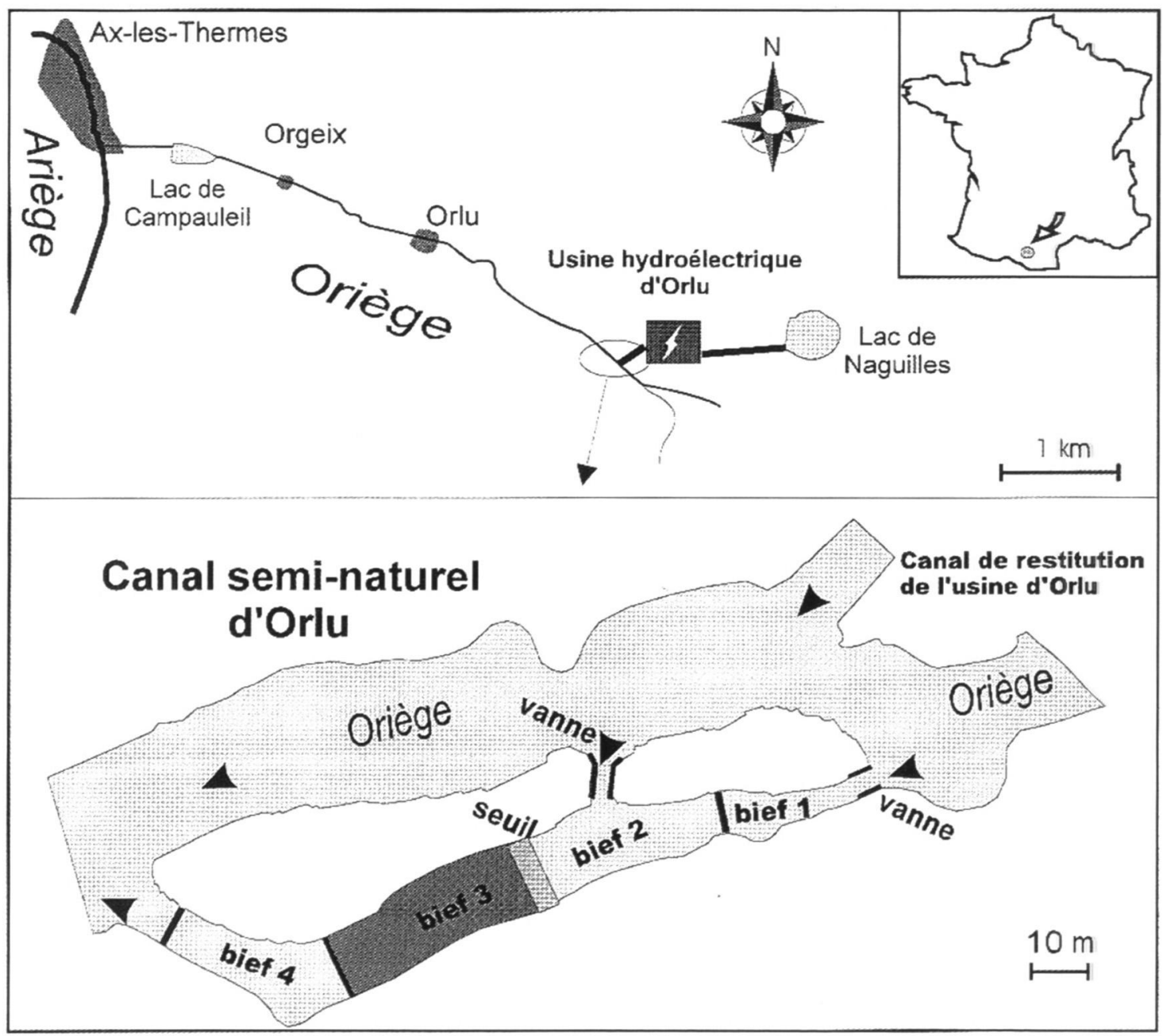

\section{Figure 1}

Localisation et configuration du canal semi-naturel d'Orlu. Le bief 3, utilisé dans cette étude, est figuré en grisé.

\section{Figure 1}

Site location and layout of the Orlu's semi-natural stream. The third reach used in this study is pointed by dark grey. 


\section{Déroulement de l'expérimentation}

Quatre modalités d'éclusées (4 séries) ont été testées (Tableau 1). Elles ont été réalisées avec 2 valeurs de débit de base (150 et $250 \mathrm{l} / \mathrm{s})$, un débit d'éclusée $(1500 \mathrm{l} / \mathrm{s})$ proche du débit plein bord du canal et 2 durées d'éclusées. Ces valeurs reconstituent dans le canal les situations qui se produisent lors d'une éclusée dans l'Oriège.

\section{Tableau I}

Détails des modalités d'éclusées testées.

Table I

Characteristics of simulated spates.

\begin{tabular}{|c|c|c|c|c|}
\hline Séries & $\begin{array}{c}\text { Débit de base } \\
\text { (Vs) }\end{array}$ & $\begin{array}{c}\text { Débit éclusée } \\
(\mathrm{V} / \mathrm{s})\end{array}$ & Accroissement & $\begin{array}{c}\text { Durée de l'éclusée } \\
\text { (minutes) }\end{array}$ \\
\hline 1 & 150 & 1500 & $\times 10$ & 10 \\
\hline 2 & 250 & 1500 & $\times 6$ & 10 \\
\hline 3 & 150 & 1500 & $\times 10$ & $>180$ \\
\hline 4 & 250 & 1500 & $\times 6$ & $>180$ \\
\hline
\end{tabular}

Les simulations ont été menées de jour sur le bief 3 qui présente un type d'écoulement mixte, radier-plat. II est doté d'un aménagement particulier associant 2 portes à guillotine à deux filets de maille $2 \mathrm{~mm}$. La limite amont du bief 3 est constituée par la dalle de béton du seuil jaugé. Un observateur posté sur la rive a vérifié qu'à aucun moment, aucun alevin ne franchissait cet obstacle.

Chaque modalité testée correspond à une série de 2 séquences d'éclusées. Pour les modalités à éclusée courte, chacune des séquences comprend 4 phases : (1) introduction d'un lot d'alevins ; (2) phase d'acclimatation ; (3) comptage des dérivants après une période de débit de base et (4) comptage des dérivants après une éclusée. Les phases 3 et 4 sont répétées plusieurs fois, mais au cours de cette étude, nous avons considéré uniquement les résultats des 2 premières éclusées de chaque séquence. Entre chaque éclusée, un retour au débit de base est systématiquement assuré afin que les alevins retrouvent un comportement " hors éclusée ". La durée de cette période est évaluée par un observateur qui, depuis la berge, détermine le moment où les alevins reprennent leurs activités (chasse, agressivité...). Entre chaque séquence, les alevins restants sont éliminés par pêche électrique et assec du bief.

Le mode opératoire est modifié pour les essais de longue durée : grâce aux 2 portes à guillotine, les 2 filets sont relevés alternativement, sans interrompre l'éclusée en cours (Figure 2). Le premier relevé est effectué après 2 minutes, suivi de 4 relevés séparés par des intervalles de 5 minutes, puis 4 autres à 10 minutes et enfin 4 à 30 minutes. Cette succession de relevés permet d'étudier la cinétique de dérive en éclusée.

Les alevins utilisés sont issus d'oeufs provenant de géniteurs sauvages prélevés à cet effet. Les oeufs, transportés dès la fécondation, sont incubés dans un petit affluent de l'Oriège où ils subissent les conditions du milieu naturel. Les alevins sont retirés au moment de l'émergence. La longueur totale moyenne mesurée sur un sous-échantillon de 30 individus par séquence expérimentale est de $25,3 \mathrm{~mm}(\mathrm{~N}=240, \sigma=1,567)$. Les différents lots d'alevins ne présentent pas de différence significative de taille (test $t$ de Student, $p=0,777$ ). 


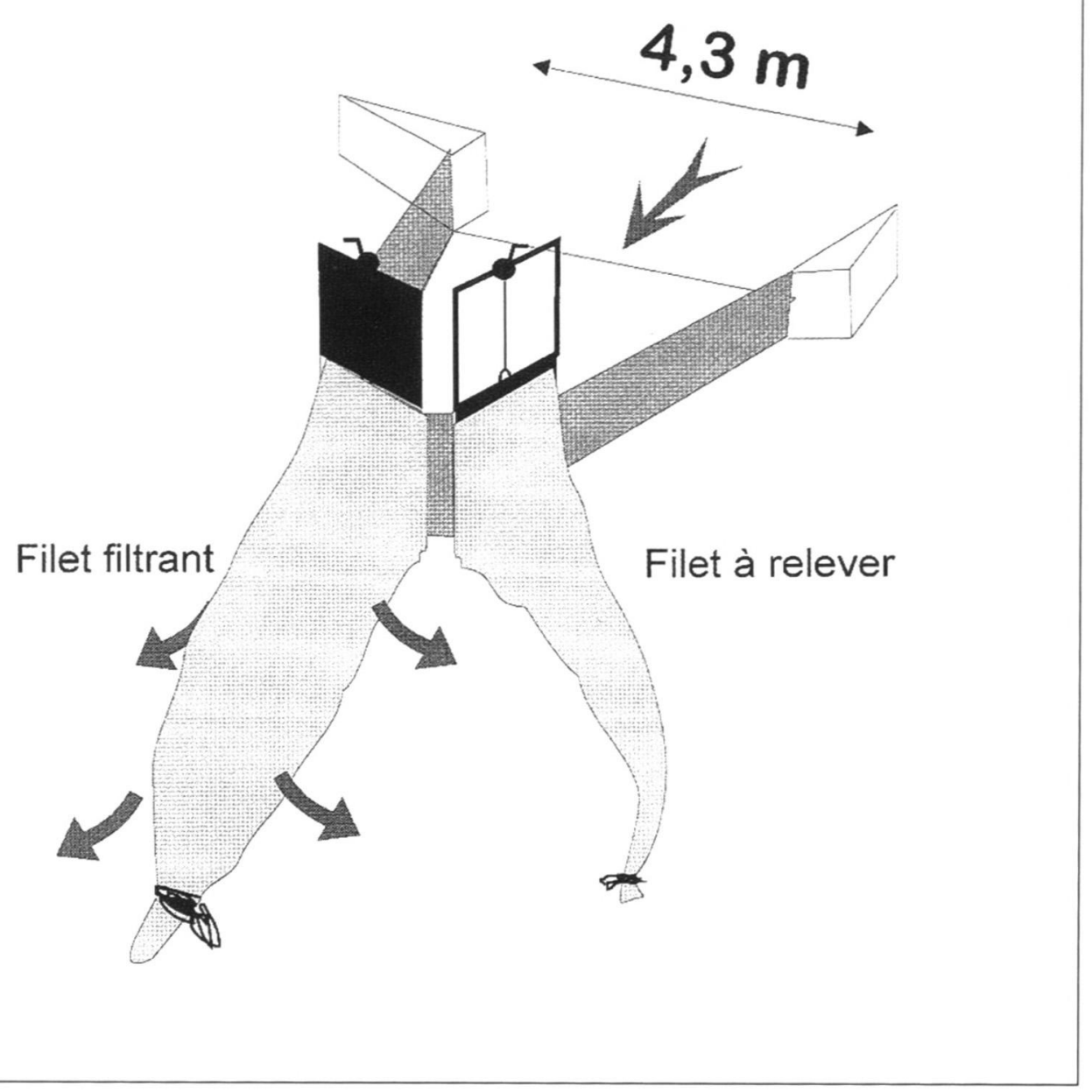

Figure 2

Schéma de l'agencement particulier des filets du bief 3 permettant de contrôler la dérive sans interrompre l'éclusée en cours.

\section{Figure 2}

Special nets used on reach 3 to control the fry drift during the spate flow.

Ces alevins sont répartis sur l'ensemble du bief en évitant la proximité immédiate du filet aval. La densité moyenne utilisée pour tous les lots est de 7,1 ind. $/ \mathrm{m}^{2}$.

Une période d'acclimatation de 15 heures et comprenant une phase nocturne est respectée avant chaque séquence d'éclusées. Les alevins ayant dérivé au cours de la phase d'acclimatation ne sont pas pris en compte, leur dévalaison étant vraisemblablement due au stress de l'installation. Pour cette raison, ils ne sont pas remis dans le bief (des tests portant sur une durée d'acclimatation plus longue, de l'ordre de plusieurs jours, n'ont montré aucune augmentation notable de cette dérive induite par le stress de l'installation). II est à noter qu'au cours des expérimentations seuls quelques cas isolés et négligeables de mortalité ont été enregistrés.

La dérive des alevins est exprimée par le taux de dérive (TD) :

TD $(\%)=$ nombre d'alevins récoltés dans les filets / nombre d'alevins initial * 100 
Les tests statistiques ont été réalisés avec la version 7.0 du logiciel SPSS (NORUSIS, 1993). La fréquence de distribution des taux de dérive ne suivant pas une loi normale (test de Lilliefors, $p=0,013$ ), les comparaisons entre modalités ont été effectuées avec le test non paramétrique de Kruskall-Wallis. Par la suite, pour déterminer la différence entre 2 groupes, le test $U$ de Mann-Whitney a été effectué sur les données regroupées selon 2 modalités, soit sur le critère de la durée des éclusées : longue / courte, soit sur celui du débit de base : fort / faible.

\section{RÉSULTATS}

\section{Dérive hors éclusées}

Au cours des phases d'acclimatation, les TD relevés variaient de 4 à $11 \%$. Cependant, du fait des divers stress occasionnés lors de la mise en place des alevins dans le bief (transport, manipulations, changement de milieu...), ils n'ont pas été intégrés aux résultats.

Par contre, lors des périodes de débit de base, et dans les conditions expérimentales testées, les alevins post-émergents n'ont manifesté aucun mouvement de dévalaison pour l'ensemble des essais.

\section{Dérive pendant éclusées}

Aucune différence significative n'est trouvée entre 2 tests d'éclusée consécutifs (test de Wilcoxon sur 8 paires, $p=0,093$ ). Notre choix d'utiliser seulement les résultats des 2 premières éclusées nous aftranchit ainsi de l'influence d'un éventuel " apprentissage " des alevins.

En revanche, les 4 types d'expérimentations montrent des résultats de dérive significativement différents (test de Kruskall-Wallis, $p<0,05$ ). Les valeurs du TD pour les 4 modalités sont données dans le Tableau II.

Du fait du nombre relativement faible de valeurs de TD obtenues, en raison des difficultés expérimentales liées notamment aux quantités d'alevins utilisées et à la gestion des débits, il n'a pas été possible de réaliser les tests statistiques par modalité. Les effets des éclusées ont donc été examinés selon le critère de durée, puis de débit de base.

\section{Tableau II}

Résultats de dérive pour les quatre modalités testées, exprimés en \% d'alevins comptés dans les filets et ramenés au nombre d'alevins initial. Les essais 1 et 2 correspondent à la première séquence expérimentale, les essais 3 et 4 à la deuxième.

\section{Table II}

Drift results for the four tested forms, expressed in \% of fry counted by nets divided by the initial number of fry. Trials 1 and 2 correspond to the first experimental sequence, trials 3 and 4 to the second one.

\begin{tabular}{ccccccc}
\hline Séries & $\begin{array}{c}\text { Durée éclusée } \\
\text { (minutes) }\end{array}$ & $\begin{array}{c}\text { Débit de base } \\
(\mathrm{Vs})\end{array}$ & Essai 1 & Essai 2 & Essai 3 & Essai 4 \\
\hline 1 & 10 & 150 & 9,91 & 9,31 & 10,03 & 9,33 \\
2 & 10 & 250 & 5,29 & 5,33 & 5,35 & 5,33 \\
3 & $>180$ & 150 & 11,81 & 12,03 & 11,70 & 11,20 \\
4 & $>180$ & 250 & 6,10 & 6,04 & 6,49 & 6,21 \\
\hline
\end{tabular}


Ainsi, si l'on considère l'effet de la durée des éclusées indépendamment de la valeur du débit de base, les TD relevés ne sont pas significativement différents (test $U$ de Mann-Whitney, $p>0,05)$. A l'opposé, en considérant un impact global des éclusées (indépendamment de la durée), le taux de dérive après un débit de base faible $(150 \mathrm{l} / \mathrm{s})$ est très significativement différent du TD après un débit de base élevé $(250 \mathrm{l} / \mathrm{s})$, (test $U$ de Mann-Whitney, $p<0,01$ ). Dans ce cas, le TD à débit de base élevé est pratiquement réduit de moitié $(-47 \%)$ par rapport au TD à débit de base faible.

\section{Cinétiques de dérive}

Les relevés intermédiaires de dévalants, réalisés sur les modalités de longue durée, permettent d'aborder l'aspect cinétique de la dérive, indépendamment de l'aspect quantitatif. La proportion d'alevins ayant dévalé par pas de temps de relevé de filet et relativement à la dévalaison totale est présentée en Figure 3 . Nous voyons ainsi que la dérive est massive en début d'éclusée : dans tous les cas, on enregistre au moins les deux tiers de la dévalaison dans les 2 premières minutes. De plus, on note une différence de $+21 \%$ pour les essais à débit de base fort $(87 \%$ de dévalants à $250 \mathrm{l} / \mathrm{s}$ contre $66 \%$ à $150 \mathrm{l} / \mathrm{s}$ ), ce qui indique une plus grande brutalité de l'effet dans ces conditions.

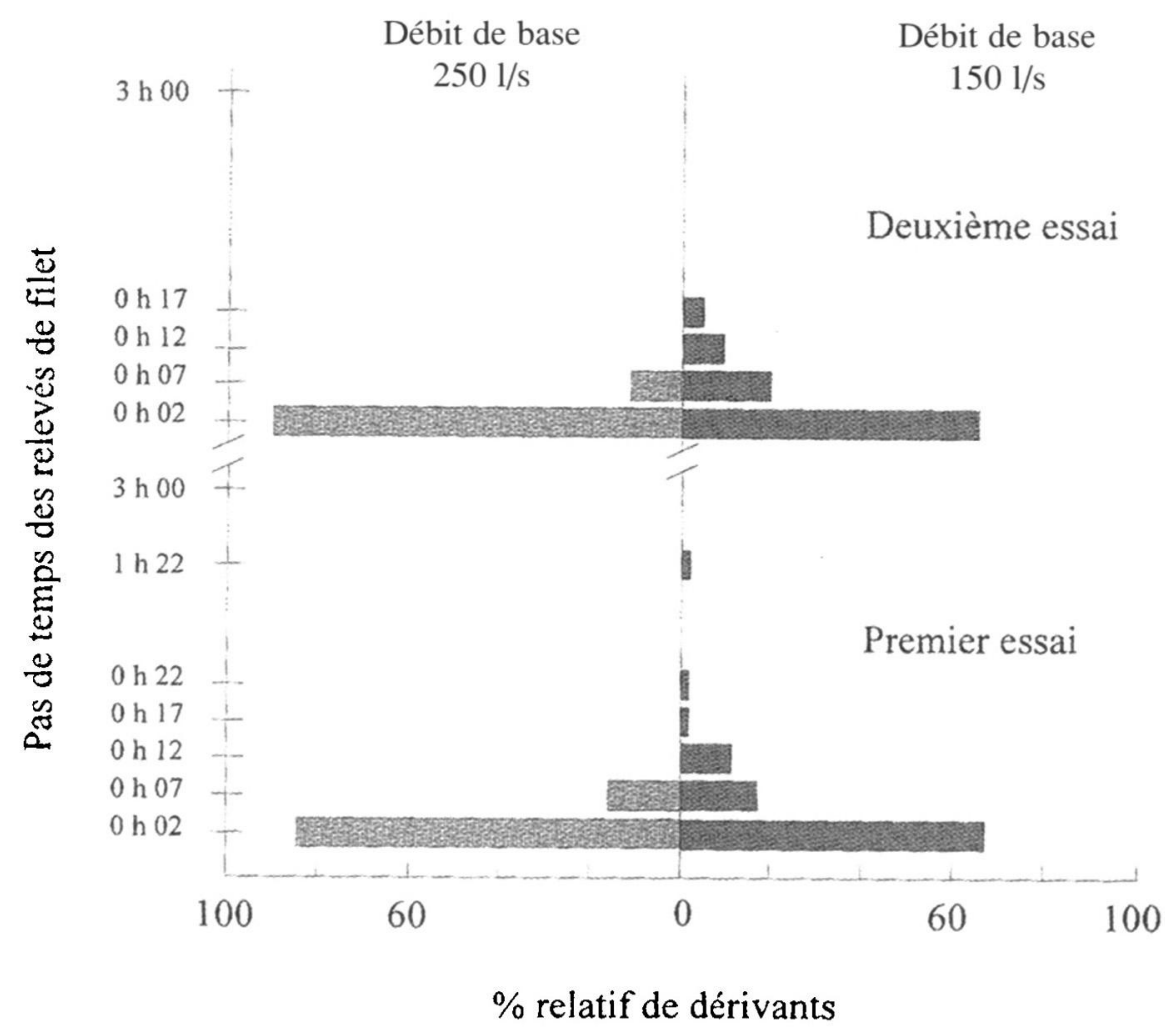

\section{Figure 3}

Exemple de cinétique de dérive, présenté pour 2 essais consécutifs de longue durée ( 3 heures), à 2 débits de base différents. Les résultats sont exprimés en pourcentage de dérivants relativement au total récoltés pour 1 essai. Seuls les relevés non-nuls ont été figurés.

\section{Figure 3}

Example of drift kinetic for two consecutive long duration trials ( 3 hours), at two different basic flows. Results are expressed in \% of drifting fry relatively to the total of the whole trial. Non null results are only shown. 
Après ce premier pic, les cinétiques de dérive présentent une diminution très rapide du pourcentage relatif d'alevins dévalants. Ainsi au bout de 7 minutes, $85 \%$ des dévalants ont été piégés dans les filets au débit de base $150 \mathrm{l} / \mathrm{s}$, et $100 \%$ au débit de base $250 \mathrm{l} / \mathrm{s}$. Après 12 minutes, $95 \%$ des alevins dévalants ont été récupérés à $150 \mathrm{l} / \mathrm{s}$.

\section{DISCUSSION}

Chez les salmonidés, la dévalaison constitue un élément clé de la régulation des populations dans le milieu naturel (HELAND, 1991), que les “ dévalants " soient considérés comme perdus (ELLIOTT, 1987) ou susceptibles de coloniser les secteurs aval (BEALL et al., 1994). Au cours de notre étude, nous n'avons enregistré aucune dérive pendant les essais correspondant à une situation naturelle (essais sur débits de base de 150 et $250 \mathrm{l} / \mathrm{s}$ ). Ces résultats sont à rapprocher de ceux de LAUTERS (1995) qui trouve, sur des alevins de truite de 36 à $47 \mathrm{~mm}$ de longueur totale, des taux de dérive compris entre 0 et $1,1 \%$ pour 10 minutes. Cette dérive très réduite est à relier aux conditions expérimentales utilisées. Nous avons travaillé en effet sur des alevins post-émergents, probablement après la période de dévalaison précoce qui intervient dans les premiers jours suivant l'émergence (HELAND, 1980). De plus, la dévalaison naturelle des jeunes salmonidés est fondamentalement nocturne (BARDONNET et GAUDIN, 1990 ; HELAND, 1991), alors que tous nos essais se sont déroulés de jour. Enfin, l'absence de prédateurs ou de compétiteurs pré-installés, qu'il s'agisse d'individus émergés précocement, de poissons plus âgés ou d'autres espèces (GAUDIN et HELAND, 1984 ; GREENBERG, 1994 ; HELAND et al., 1995), est certainement impliquée dans l'absence de dévalaison naturelle.

A l'opposé, en situation perturbée par des éclusées, l'existence d'une dérive provoquée des alevins post-émergents est clairement montrée. Ce phénomène touche à chaque évènement, de 5 à $12 \%$ des individus présents. Rapprochés des observations de VALENTIN (1995) qui relève peu d'impacts des éclusées sur la dérive d'alevins de truite en canal expérimental, mais dans des conditions sensiblement différentes, impliquant notamment des individus plus âgés ( 2 mois), des densités beaucoup plus faibles ( 1 ind. $\left./ \mathrm{m}^{2}\right)$ et des éclusées à débit réduit $(300 \mathrm{l} / \mathrm{s})$, ces résultats soulignent la pertinence du choix du matériel biologique (alevins post-émergents) et l'importance de refléter l'aspect "catastrophique " des éclusées. Nous pouvons alors penser que le volume des éclusées que nous avons simulées $(1500 \mathrm{l} / \mathrm{s})$ associé à l'hétérogénéité du milieu naturel (GREENBERG, 1994) conservée au canal, et au choix des alevins, contribuent à se rapprocher des situations rencontrées par les alevins dans une rivière de tête de bassin soumise à une gestion par éclusées, validant ainsi nos résultats.

II est aussi clair que les éclusées n'ont pas toutes eu le même impact. Certains facteurs comme le débit de base peuvent influer sur l'ampleur de la dérive (LAUTERS, 1995 ; VALENTIN, 1995). Ainsi, nous avons mis en évidence une diminution du taux de dérive de $47 \%$, lorsque ce débit de base est fixé à une valeur élevée (250 l/s comparé à $150 \mathrm{l} / \mathrm{s})$. Nous pouvons expliquer ce résultat par le comportement de l'alevin après l'émergence. II est communément admis que pendant cette période, l'alevin recherche individuellement des postes de chasse où la dépense énergétique est minimale (abris face au courant), pour un gain maximal (accès privilégié à la dérive d'invertébrés) (FAUSCH, 1984 ; HELAND, 1991). Soumis à un débit soutenu, l'alevin tend à occuper des postes plus abrités et se rapproche notamment du substrat où les conditions hydrauliques sont plus favorables (VALENTIN, 1994b), des réenfouissements dans le substrat étant même rapportés en réponse à des variations de débit (VALENTIN, 1995). De ce fait, lorsque l'éclusée se produit, l'alevin, qui se trouve déjà à proximité immédiate d'un abri hydraulique important, est moins susceptible d'être entraîné. D'autre part, si l'on considère l'influence 
de la durée de l'éclusée sur la dérive (LAUTERS, 1995), celle-ci n'est pas constante. La dérive diminue rapidement, l'impact principal se produisant pendant la montée de débit (effet vague). Quel que soit le débit de base considéré, nous avons enregistré $95 \%$ à $100 \%$ de la dérive totale dans les 12 premières minutes de l'éclusée, valeurs à rapprocher des résultats de VALENTIN (1994b) qui ne capture d'ombres dévalants que dans les 10 premières minutes. Les éclusées de longue durée induisent ainsi une intensité de dérive proche de celle des éclusées de courte durée.

Cependant, à débit de base élevé, nous avons observé dans les 2 premières minutes de l'éclusée, $21 \%$ de dévalants de plus qu'à débit faible. II est probable que cette différence d'intensité de dérive, qui joue sur la brutalité de l'effet et non pas sur la quantité d'alevins touchés, est le fait d'alevins incapables de s'intégrer à une mosaïque territoriale (HELAND, 1991) rendue plus rigide par des conditions de débit relativement défavorables (250 l/s par rapport à $150 \mathrm{l} / \mathrm{s}$ ). Ces individus sans territoire, donc directement exposés, sont emportés dès le début de l'éclusée. Au contraire, les alevins qui ont eu la possibilité de rejoindre un refuge sont à l'abri et ne participent pas à cette dérive. L'absence d'alevins dans les filets pendant les heures suivantes tend à confirmer cette hypothèse.

\section{CONCLUSION}

En définitive, il apparaît que les populations d'alevins de truite sont dépendantes, dès l'émergence, de variables abiotiques liées à la gestion par éclusées des usines hydroélectriques. A l'aval de ces ouvrages, une dérive forcée de forte ampleur s'ajoute à la dévalaison naturelle qui intervient avec la mise en place de comportements sociaux et de sélection ou d'occupation d'habitat. L'ampleur du protocole expérimental mis en place, conjointement à la brièveté de la période utilisable (stade de développement des alevins et conditions de débit), ont limité les résultats à deux facteurs : le rôle du débit de base et de la phase de démarrage sur l'importance de la dérive. Néanmoins, la mise en évidence de leur action sur les effets des éclusées apporte des éléments de réflexion aux gestionnaires des centrales hydroélectriques, permettant d'envisager un compromis production d'énergie/protection des alevins. A ce titre, dans l'estimation de l'impact des éclusées, on doit nécessairement tenir compte de la répétitivité de ces perturbations hydrauliques. Les effets des 2 premières éclusées peuvent s'additionner simplement (10 à $24 \%$ du stock touché), mais pour les suivantes, on doit envisager un amortissement par " apprentissage " des alevins, voie qui reste à explorer. Par ailleurs, la fragilité particulière des alevins post-émergents par rapport aux individus plus âgés ( 1 à 2 mois) va permettre d'ajuster la gestion aménagée des usines à une période de sensibilité en définitive assez réduite mais qu'il conviendra de déterminer.

Enfin, ces résultats soulignent l'efficacité du canal semi-naturel de l'Oriège qui, en conditions réelles, a répondu par des signaux clairs aux différentes simulations réalisées. La fiabilité de ce système permettra de tester de nouvelles hypothèses, telles que l'influence sur la dérive des alevins de la densité d'individus ou du nombre d'éclusées supportées (apprentissage), et de compléter cette étude par une approche plus déterministe, basée notamment sur l'analyse des fluctuations locales des paramètres du microhabitat.

\section{REMERCIEMENTS}

L'aménagement du canal expérimental a pu être réalisé grâce à l'aide financière fournie par EDF (Direction des Etudes et Recherches) et à l'aimable autorisation de I'AAPPMA d'Orlu. 


\section{BIBLIOGRAPHIE}

BARDONNET A., GAUDIN P., 1990. Diel pattern of post-emergence displacement in grayling Thymallus thymallus (L., 1758). J. Fish. Biol., 37, 623-627.

BARDONNET A., GAUDIN P., THORPE J.E., 1993. Diel rhythm of emergence and of first displacement downstream in trout (Salmo trutta), Atlantic salmon (S. salar) and grayling (Thymallus thymallus). J. Fish. Biol., 19, 755-762.

BEALL E., DUMAS J., CLAIREAUX D., BARRIERE L., MARTY C., 1994. Dispersal patterns and survival of Atlantic salmon (Salmo salar L.) juveniles in a nursery stream. ICES J. Mar. Sci., 51, 1-9.

CEREGHINO R., 1997. Influence des éclusées hydroélectriques sur la structure et la dynamique des populations d'invertébrés d'une rivière pyrénéenne de moyenne montagne. Thèse 3è cycle, Université Paul Sabatier de Toulouse, p. 175.

CUSHMAN R.M., 1985. Review of ecological effects of rapidly varying flows downstream from hydroelectrics facilities. North Am. J. Fish. Manag., 5, 330-339.

ELLIOTT J.M., 1987. The distances travelled by downstream-moving trout fry, Salmo trutta, in a Lake District stream. Freshwater Biology, 17, 491-499.

ELLIOTT J.M., 1989. The critical-period concept for juvenil survival and its relevance for population regulation in young sea trout, Salmo trutta. J. Fish. Biol., 35, 91-98.

FAUSCH K.D., 1984. Profitables stream positions for salmonids relating specific growth rate to net energy gain. Can. J. Zool., 62, 441-451.

GAUDIN P., HELAND M., 1984. Influence d'adultes de chabots (Cottus gobio L.) sur des alevins de truite commune (Salmo trutta L.) : étude expérimentale en milieux seminaturels. Acta Oecologica, Oecol. applic., 5, 71-83.

GAUDIN P., HELAND M., 1995. Stratégies d'utilisation de l'habitat par des alevins postémergents de truite commune (Salmo trutta) et de saumon atlantique (Salmo salar). In GAUDIN P., SOUCHON Y., ORTH D.J., VIGNEUX E. : Colloque " HabitatPoissons ", 1995. Bull. Fr. Pêche Piscic., 337/338/339, 418 p.

GREENBERG L.A., 1994. Effects of predation, trout density and discharge on habitat use by brown trout, Salmo trutta, in artificial streams. Freshwater Biology, 32, 1-11.

HEGGENES J., TRAAEN T., 1988. Downstream migration and critical water velocities in streams channels for fry of four salmonids species. J. Fish. Biol., 32, 717-727.

HELAND M., 1980. La dévalaison des alevins de truite commune Salmo trutta L. I. Caractérisation en milieu artificiel. Ann/s Limnol., 16, 233-245.

HELAND M., 1991. Organisation sociale et territorialité chez la truite commune immature au cours de l'ontogenèse. In BAGLINIĖRE J.L. et MAISSE G., La truite, biologie et écologie, INRA publ., Paris, 121-149.

HELAND M., GAUDIN P., BARDONNET A., 1995. Mise en place des premiers comportements et utilisation de l'habitat après l'émergence chez les saimonidés d'eau courante. In GAUDIN P., SOUCHON Y., ORTH D.J., VIGNEUX E. : Colloque " Habitat-Poissons », 1995. Bull. Fr. Pêche Piscic., 337/338/339, 418 p.

IRVINE J.R., 1986. Effects of varying discharge on the downstream movement of salmon fry, Oncorhynchus tshawytscha Walbaum. J. Fish. Biol., 28, 17-28.

LAUTERS F., 1992. Impact sur l'écosystème rivière de la gestion par éclusées des ouvrages hydroélectriques : étude bibliographique. EDF/DER, HE31/92-09, p. 42.

LAUTERS F., 1993. La gestion par éclusées des ouvrages hydroélectriques d'Electricité De France : enquête auprès des exploitants. EDF/DER, HE31/92-19, p. 102.

LAUTERS F., 1995. Impacts sur l'écosystème aquatique de la gestion par éclusées des ouvrages hydroélectriques. Thèse 3è cycle, Université Paul Sabatier de Toulouse, p. 188. 
NORUSIS M.J., 1993. SPSS for Windows, Base system Users guide, release 7.0. SPSS Inc., p. 828.

OTTAWAY E.M., CLARKE A., 1981. A preliminary investigation into the vulnerability of young trout Salmo trutta $L$. and Atlantic salmon $S$. salar $L$. to downstream displacement by high water velocities. J. Fish. Biol., 19, 135-145.

VALENTIN S., SOUCHON Y., WASSON J.G., 1994a. Evaluation of hydropeaking effects on fish community and habitat. In COWX I.G., Rehabilitation of Freshwater Fisheries, Fishing News Books, Blackwell Scientific Publications, Oxford, 138-151.

VALENTIN S., SEMPESKI P., SOUCHON Y., GAUDIN P., 1994b. Short-term habitat use by young grayling, Thymallus thymallus $L$., under variable flow conditions in an experimental stream. Fisheries Management and Ecology, 1, 57-65.

VALENTIN S., 1995. Variabilité artificielle des conditions d'habitat et conséquence sur les peuplements aquatiques : effets écologiques des éclusées hydroélectriques en rivière. Thèse 3è cycle, Université Claude Bernard Lyon 1, p. 275. 